\title{
THE LEPTON MIXING MATRIX IN A COMPOSITE MODEL
}

\author{
Yukio TOMOZAWA \\ Randall Laboratory of Physics, University of Michigan, Ann Arbor, MI 48109, USA
}

Received 13 May 1981

\begin{abstract}
A generalized mixing matrix for leptons is presented based on a composite model of quarks and leptons. The mixing matrix is expressed in terms of one parameter, which is determined either by discussing that it is identical to that of the quark mixing matrix or by assuming that the observed solar neutrino flux results from neutrino oscillations.
\end{abstract}

The problem of generations of quarks and leptons may be viewed as indicative of a new symmetry group (the horizontal group), or of a composite model [1]. Despite some difficulties in the models, which are characteristic for an infant stage of research, in particular the question of the mass spectrum, both directions have been pursued vigorously in the last few years.

In a composite model of quarks and leptons, the generations are considered to be radial excited states with zero angular momentum. Assuming that it is a bound-state problem of two-body systems, several authors [2-4] proposed a method to calculate the mixing matrix (thereafter called the $U$-matrix). Recently the author [5] and Akama and Terazawa [6] independently calculated the $U$-matrix for various potentials and compared the result with the available experimental data.

(a) The quark mixing matrix. The authors assumed the potential is energy and angular-momentum dependent and is divided into two parts,

$V(r, E, l)=V(r)+V_{l}(E)$,

a local potential $V(r)$ and the $r$ independent but $E$ and $l$ dependent part $V_{l}(E)$. By this assumption, the author dissociates the question of the mass spectrum and the nonappearance of orbital angular momentum excited states [5]. Then the $U$-matrix elements for quarks are expressed as overlap integrals of the wave functions of the up-quark series and the down-quark series. It is shown that the result depends on one parameter which is determined by the experimental value of the Cabibbo angle. Among the potentials used (a harmonic oscillator, the Coulomb, and a rigid wall potential), a harmonic oscillator potential gives the most satisfactory resuit. [For a potential $V(r)=a r^{s}$, the suggested value of the exponent is [5] $\left.s=2_{-2}^{+4}\right]$. For simplicity, I will use a harmonic oscillator potential

$V(r)=\frac{1}{2} m \omega^{2}-r^{2}$

The s-state wave functions are given by [7]

$R_{n}(r, k)=(-1)^{n-1}\left\{2 \Gamma\left(n+\frac{1}{2}\right) k^{3} / \Gamma(n)\left[\Gamma\left(\frac{3}{2}\right)\right]^{2}\right\}^{1 / 2} \exp \left(-\frac{1}{2} \rho^{2}\right) F\left(-(n-1) ; \frac{3}{2} ; \rho^{2}\right), \quad n=1,2,3 \ldots$,

where

$\rho=k r=\sqrt{m \omega} r$,

and $F(a ; b ; z)$ is the confluent hypergeometric function. Assuming that the up-quark and the down-quark system have different $k$ values ( $k$, and $k^{\prime}$, respectively), the matrix element $a_{n l}$ of the $U$-matrix is then given by [8] 


$$
\begin{aligned}
a_{n l} & =\int_{0}^{\infty} R_{n}(r, k) R_{l}\left(r, k^{\prime}\right) r^{2} \mathrm{~d} r \\
& =(-1)^{n-1}\left(\frac{(2 n-1) ! !(2 l-1) ! !}{(2 n-2) ! !(2 l-2) ! !}\right)^{1 / 2}(\sin \theta)^{3 / 2}(\cos \theta)^{n+l-2} F\left(-(n-1),-(l-1) ; \frac{3}{2} ;-\tan ^{2} \theta\right)
\end{aligned}
$$

where

$\sin \theta=2 z /\left(1+z^{2}\right), \quad \cos \theta=\left(1-z^{2}\right) /\left(1+z^{2}\right), \quad z=k / k^{i}=\tan \frac{1}{2} \theta$

and $F(a, b ; c ; z)$ is the hypergeometric function. The explicit form of $a_{n l}(n, l \leqslant 4)$ is given in ref. [5]. From eq.

(5) and (6), follow the symmetry properties

$a_{n l}(\theta)=a_{l n}(\pi-\theta)=(-1)^{n+l} a_{l n}(\theta)$.

The $U$-matrix is obviously an orthogonal matrix since the solution (3) is an orthonormal complete set. We notice that $a_{1 l}$ has the simplest form and is given by

$a_{1 l}=[(2 l-1) ! ! /(2 l-2) ! !]^{1 / 2}(\sin \theta)^{3 / 2}(\cos \theta)^{l-1}, \quad l=1,2 \ldots$

It obviously satisfies the condition

$\sum_{l=1}^{\infty} a_{1 l}^{2}=(\sin \theta)^{3} \sum_{l=1}^{\infty} \frac{(2 l-1) ! !}{(2 l-2) ! !}(\cos \theta)^{l-1}=(\sin \theta)^{3}\left(1-\cos ^{2} \theta\right)^{-3 / 2}=1$.

Equating

$a_{12}=(3 / 2)^{1 / 2}(\sin \theta)^{3 / 2} \cos \theta$

to the experimental value of the Cabibbo angle

$\sin \theta_{c}=0.219$,

we obtain

$\sin \theta=0.983 \quad$ (or $z=\tan \frac{1}{2} \theta=0.931$ ).

This leads to the determination of the $U$-matrix for quarks

$U=\left(\begin{array}{ccccc}0.9746 & \underline{0.219} & 0.0450 & 0.00892 & \ldots \\ -0.219 & 0.892 & 0.376 & 0.113 & \ldots \\ 0.0450 & -0.376 & 0.753 & 0.495 & \ldots \\ -0.00892 & 0.113 & -0.495 & 0.571 & \ldots \\ . & . & . & . & \ldots\end{array}\right)$

These should be compared with the values obtained from the experimental data [9]

$\left|a_{11}\right|=0.9737 \pm 0.0025, \quad\left|a_{12}\right|=0.219 \pm(0.002 ; 0.011) \quad\left|a_{13}\right|=0.06 \pm 0.06$,

and [10]

$$
\text { (input) }
$$


$0.192<\left|a_{21}\right|<0.34, \quad\left|a_{22}\right|=0.8 \pm 0.2, \quad\left|a_{23}\right|>0.01$

(b) The observed solar neutrino flux. Studies of the solar neutrino flux indicate that the observed flux is $0.25-0.38$ times the theoretical value expected from the standard model of the nuclear reaction in the sun [11]. This may not be definitive evidence for neutrino oscillations, since a subtle change of the solar model could change the expected solar neutrino flux.

Let us calculate the depletion rate of the solar neutrino flux due to neutrino oscillations defined by $I=\frac{\text { (the solar neutrino flux with neutrino oscillation) }}{\text { (the expected value without neutrino oscillation) }}$.

The value of $I$ is obtained [12] from the U-matrix element for leptons, the neutrino masses $m_{1}, m_{2}, \ldots$, the energy $E$ of $\nu_{\mathrm{e}}=a_{11} \nu_{1}+a_{12} \nu_{2}+\ldots$ and the distance between the sun and the earth, $L$;

$$
I \equiv P\left(\nu_{\mathrm{e}} \rightarrow \nu_{\mathrm{e}}\right)
$$

$$
=\left.\left.\left|\sum_{n=1}^{\infty}\right| a_{1 n}\right|^{2} \exp \left(-\frac{1}{2} \mathrm{i} m_{n}^{2} L / E\right)\right|^{2}=\sum_{n=1}^{\infty}\left|a_{1 n}\right|^{4}+2 \sum_{n \neq l}\left|a_{1 n}\right|^{2}\left|a_{1 l}\right|^{2} \cos \frac{1}{2}\left(m_{n}^{2}-m_{l}^{2}\right) L / E \rightarrow \sum_{n=1}^{\infty}\left|a_{1 n}\right|^{4} .
$$

Here we have assumed that all neutrino masses are smaller than $1 \mathrm{MeV}$ and $m_{n}^{2}-m_{l}^{2} \gg\left(10^{-6} \mathrm{eV}\right)^{2}$ so that the argument of $\cos$ in the above equation is a large number. [Averaging over $L$ makes the oscillating term vanish in this case.] From eq. (8), it follows that ${ }^{\neq 1}$

$$
\begin{aligned}
I & =\sum_{n=1}^{\infty}\left|a_{1 n}\right|^{4}=\sum_{n=0}^{\infty} \frac{(2 n+1) ! !}{(2 n) ! !}(\cos \theta)^{4 n}(\sin \theta)^{6} \\
& =F\left(\frac{3}{2}, \frac{3}{2} ; 1 ; \cos ^{4} \theta\right)(\sin \theta)^{6}=(2 / \pi)\left[\sin ^{2} \theta /\left(1+\cos ^{2} \theta\right)\right] E\left(\left[2 \cos \theta /\left(1+\cos ^{2} \theta\right)\right]^{2}\right),
\end{aligned}
$$

where

$$
E(m)=\int_{0}^{\pi / 2}\left(1-m \sin ^{2} t\right)^{1 / 2} \mathrm{~d} t
$$

is the complete elliptic integral of the second kind. Fig. 1 depicts the $\theta$ dependence of the quantity $I$. If one assumes that the $U$-matrices of quarks and leptons are identical, one obtains

$$
\begin{aligned}
I= & \left.(2 / \pi)\left[\sin ^{2} \theta /\left(1+\cos ^{2} \theta\right)\right] E\left(\left[2 \cos \theta /\left(1+\cos ^{2} \theta\right)\right]^{2}\right)\right|_{\sin \theta=0.983} \\
& =(0.9746)^{4}+(0.219)^{4}+(0.045)^{4}+(0.00892)^{4}+\ldots=0.905
\end{aligned}
$$

This value is much too large compared with the observed value

$$
I_{\text {obs }}=(0.25-0.38)=\text { average value of } 0.32 \text {. }
$$

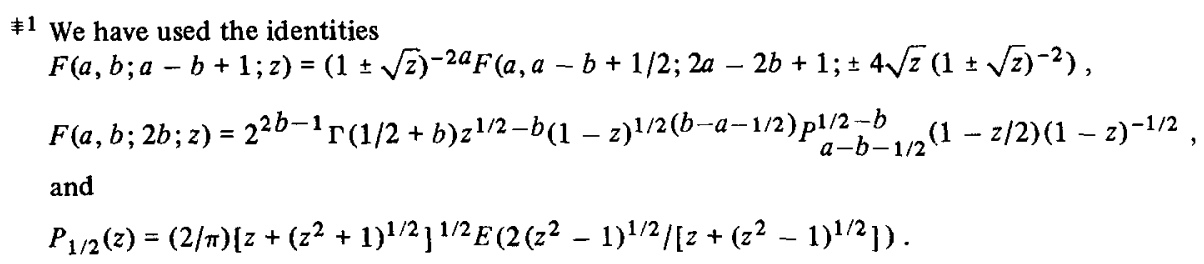

See ref. [13]. 


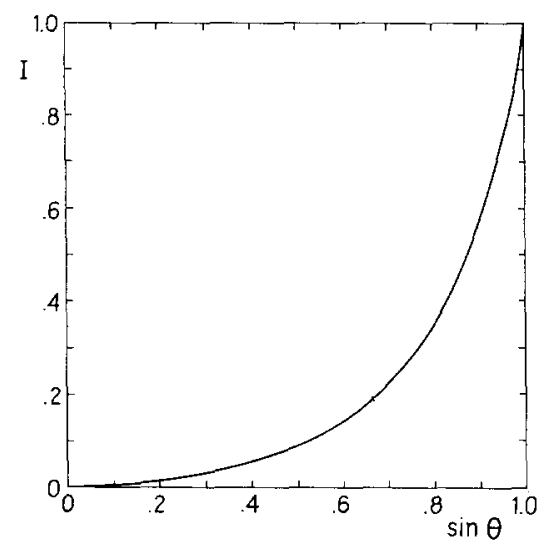

$4 \quad$ Fig. $1 . I=P\left(\nu_{\mathrm{e}} \rightarrow \nu_{\mathrm{e}}\right)$ as a function of the parameter $\theta$.

In the next subsection, assuming that the observed value of $I$ is due to neutrino oscillations we determine the $U$ matrix for leptons.

(c) An alternative choice for the lepton mixing matrix. From the equality

$I_{\mathrm{obs}} \simeq 0.32=(2 / \pi)\left[\sin ^{2} \theta /\left(1+\cos ^{2} \theta\right)\right] E\left(\left[2 \cos \theta /\left(1+\cos ^{2} \theta\right)\right]^{2}\right)$,

we obtain

$\sin ^{2} \theta=0.6 \quad(\sin \theta=0.775)$.

This gives the $U$-matrix for leptons to be

$U=\left(\begin{array}{ccccc}0.682 & 0.528 & 0.373 & 0.255 & \ldots \\ -0.528 & 0 & 0.289 & 0.395 & \ldots \\ 0.373 & -0.289 & -0.368 & -0.196 & \ldots \\ -0.255 & 0.395 & 0.196 & -0.120 & \ldots \\ . & . & . & \ldots\end{array}\right)$

The vanishing value for $a_{22}$ is the result of the expression

$a_{22}=(\sin \theta)^{3 / 2}\left(1-\frac{5}{2} \cos ^{2} \theta\right)$,

and the value chosen for the parameter $\cos ^{2} \theta=0.4$. Table 1 gives the values of the mixing matrix elements and the depletion factor $I$ of the solar neutrinos for various values of the parameter $\sin \theta$. Since the two choices for the

Table 1

The lepton mixing matrix elements $a_{n l}$ and $I$ [defined by eqs. (17) and (18)] for different values of the parameter sin $\theta$.

\begin{tabular}{ccccccccccccc}
\hline $\sin \theta$ & $I$ & $a_{11}$ & $a_{12}$ & $a_{13}$ & $a_{14}$ & $a_{12}$ & $a_{23}$ & $a_{24}$ & $a_{33}$ & $a_{34}$ & $a_{44}$ \\
\hline 0.983 & 0.905 & 0.975 & 0.219 & 0.0450 & 0.00892 & 0.892 & 0.376 & 0.113 & 0.753 & 0.495 & 0.571 \\
0.813 & $\mathbf{0 . 3 7 9}$ & $\mathbf{0 . 7 3 3}$ & 0.523 & 0.340 & 0.214 & 0.112 & 0.388 & 0.442 & -0.343 & -0.0712 & -0.260 \\
0.775 & $\mathbf{0 . 3 1 8}$ & $\mathbf{0 . 6 8 2}$ & $\mathbf{0 . 5 2 8}$ & 0.373 & 0.255 & 0 & 0.289 & 0.395 & -0.368 & -0.196 & -0.120 \\
0.720 & 0.250 & 0.611 & $\mathbf{0 . 5 1 9}$ & 0.403 & 0.302 & -0.125 & 0.149 & 0.296 & -0.333 & -0.289 & 0.0697 \\
\hline
\end{tabular}


lepton mixing matrix, eq. (13) or (23), are vastly different, we are analyzing the existing experimental data for neutrino oscillation and the $\mu \rightarrow$ e $\gamma$ experiment based on either of these choices. The result will be reported elsewhere.

It is a great pleasure to thank Vladimir Visnjic and Martinus Veltman for stimulation and encouragement and David Williams for reading the manuscript. The work is supported in part by the US Department of Energy.

\section{References}

[1] A partial list includes: K. Tennakone and S. Pakvasa, Phys. Rev. Lett. 27 (1971) 757; Phys. Rev. D6 (1972) 2494; J.C. Pati, A. Salam and J. Strathdee, Phys. Lett. 59B (1975) 265;

H. Terazawa, Y. Chikashige and K. Akama, Phys. Rev. D15 (1977) 480;

G.'t Hooft, Lecture Cargese Summer Institute (1979);

M. Veltman, Proc. 1979 Intern. Symp. on Lepton and photon interactions at high energies, eds. T.B.W. Kirk and H.D.I.

Abarbanel (Fermilab, 1979) p. 529;

Y. Ne'eman, Phys. Lett. 82B (1979) 69;

H. Harari, Phys. Lett. 86B (1979) 83;

M.A. Shupe, Phys. Lett. 86B (1979) 87;

M. Glück, Phys. Lett. 87B (1979) 247;

H.J. Lipkin, Phys. Lett. 89B (1980) 358;

K. Akama, Prog. Theor. Phys. 64 (1980) 1494;

G.L. Shaw, D. Silverman and R. Slansky, Phys. Lett. 94B (1980) 57;

P.F. Smith and J.D. Lewin, Phys. Lett. 94B (1980) 484;

H. Terazawa and K. Akama; Phys. Lett 96B (1980) 276; 97B (1980) 81;

S.J. Brodsky and S.D. Drell, SLAC-PUB-2534 (1980);

I. Bars and M. Gunaydin, Phys. Rev. D22 (1980) 1403; Phys. Lett. 95B (1980) 373;

E. Derman, Phys. Lett. 95B (1980) 369;

A. De Rújula, Phys. Lett. 96B (1980) 279;

R. Casalbuoni and R. Gatto; Phys. Lett. 90B (1980) 81.

[2] V. Visnjic-Triantafillou, Fermilab-Pub-80/34-THY (March 1980).

[3] H. Terazawa, Progr. Thecr. Phys. 64 (1980) No. 5.

[4] O.W. Greenberg and J. Sucher, Univ. of Maryland Pub. No. 81-026 (August 1980).

[5] Y. Tomozawa, UM HE 81-3 (1981).

[6] K. Akama and H. Terazawa, INS-Rep.-396 (December 1980).

[7] R.A. Buckingham, in: Quantum theory I. Elements, ed. R. Bates (Academic Press, New York, 1961).

[8] I.S. Gradshteyn and I.W. Ryzhik, Table of integrals, series and products (Academic Press, 1965), Formula 7.622.

[9] R.E. Shrock, S.B. Treiman and L.L. Wang, Phys. Rev. Lett. 42 (1979) 1589.

[10] S. Pakvasa, S.F. Tuan and J.J. Sakurai, UH-511-427-80 (1980).

[11] J.N. Bahcall et al., Phys. Rev. Lett. 45 (1980) 945.

[12] E.g. S.M. Bilenky and B. Pontecorvo, Phys. Rep. 41C (1978) 225; V. Barger et al., Phys. Rev. D22 (1980) 1636, 2718; Phys. Lett. 93B (1980) 194.

[13] M. Abramowitz and I.A. Stegun, eds., Handbook of mathematical functions (Dover, New York, 1972), (15.3.27), (15.4.7) and (8.13.5). 